\title{
ORBIFOLDS ARE NOT COMMUTATIVE GEOMETRIES
}

\author{
ADAM RENNIE ${ }^{\bowtie}$ and JOSEPH C. VÁRILLY
}

(Received 12 April 2007; revised 7 November 2007)

Communicated by G. A. Willis

\begin{abstract}
In this note we show that the crucial orientation condition for commutative geometries fails for the natural commutative spectral triple of an orbifold $M / G$.
\end{abstract}

2000 Mathematics subject classification: 57P05, 58B34.

Keywords and phrases: noncommutative geometry, spectral triple, orbifold.

\section{Introduction}

A spectral triple $(\mathcal{A}, \mathcal{H}, \mathcal{D})$ consists of a unital *-algebra $\mathcal{A}$ represented on the Hilbert space $\mathcal{H}$ as bounded operators, along with an unbounded self-adjoint operator $\mathcal{D}:$ Dom $\mathcal{D} \subset \mathcal{H} \rightarrow \mathcal{H}$ such that $[\mathcal{D}, a]$ is bounded for all $a \in \mathcal{A}$ and $\left(1+\mathcal{D}^{2}\right)^{-1 / 2}$ is a compact operator on $\mathcal{H}$.

Spectral triples generalize the analytic data associated to the index problem for elliptic first-order differential operators on manifolds. For example, if $(M, g)$ is a closed oriented Riemannian manifold, then $\left(C^{\infty}(M), L^{2}\left(M, \Lambda^{\bullet} T_{\mathbb{C}}^{*} M\right), d+d^{*}\right)$ is a spectral triple, whose Hilbert space consists of square-integrable differential forms. If $(M, g)$ carries a $\operatorname{spin}^{c}$ structure with spinor bundle $S \rightarrow M$ and Dirac operator $\not D$, one may use the Dirac spectral triple $\left(C^{\infty}(M), L^{2}(M, S), \not D\right)$ instead. More generally, spectral triples with noncommutative algebras provide the basic data in Connes' programme for noncommutative metric geometries.

Recently, in [4], it was shown that a spectral triple $(\mathcal{A}, \mathcal{H}, \mathcal{D})$ with $\mathcal{A}$ commutative (and unital) is the Dirac triple of a $\operatorname{spin}^{c}$ manifold if and only if it satisfies certain

This work was supported by an ARC grant, DP0211367, and by the Statens Naturvidenskabelige Forskningsråd, Denmark. Support from the University Complutense de Madrid and the Vicerrectoría de Investigación of the Universidad de Costa Rica is acknowledged.

(c) 2008 Australian Mathematical Society 1446-7887/08 \$A2.00+0.00 
additional conditions of an algebraic or operatorial nature. These conditions are a mild strengthening and generalization of those proposed by Connes in [2]. (A spectral triple with $\mathcal{A}$ commutative and satisfying some version of these conditions could thus be called a commutative geometry, hence the title.)

In this note we show precisely why orbifolds of the form $M / G$, with $G$ a finite group acting unfreely by isometries on $M$, fail to satisfy these conditions. For such orbifolds we can define a commutative spectral triple, and although we do not show it here, these spectral triples do satisfy the majority of those conditions. However, the crucial orientation condition, which provides a volume form, tangent bundle and ultimately gives the local coordinates, fails for the spectral triples of the orbifolds.

After a brief recollection of our conditions, we go directly to the demonstration of the failure of the orientation condition. To finish, we briefly comment on more general orbifolds.

\section{Geometric conditions for commutative spectral triples}

In [4] we have shown that under a small number of postulates, mildly extending those of [2], any spectral triple $(\mathcal{A}, \mathcal{H}, \mathcal{D})$ whose algebra $\mathcal{A}$ is commutative and unital satisfies $\mathcal{A}=C^{\infty}(M)$ where $M$ is a smooth closed manifold carrying a spin (or $\operatorname{spin}^{c}$ ) structure, $\mathcal{H}$ is its $L^{2}$-spinor space and $\mathcal{D}$ is a Dirac operator plus an endomorphism of the spinor bundle.

Recall that a (unital) spectral triple consists of a unital algebra $\mathcal{A}$ faithfully represented on a Hilbert space $\mathcal{H}$, and a selfadjoint operator $\mathcal{D}$ on $\mathcal{H}$, with compact resolvent, such that $\mathcal{A}$ preserves its domain Dom $\mathcal{D}$ and $[\mathcal{D}, a]$ extends to a bounded operator on $\mathcal{H}$ for each $a \in \mathcal{A}$.

Assume furthermore that $\mathcal{A}$ is commutative. Denote by $A$ its norm completion in $\mathcal{B}(\mathcal{H})$ and by $M=\operatorname{sp}(A)=\operatorname{sp}(\mathcal{A})$ its character space (Gelfand spectrum); here $A$ is assumed to be a separable $C^{*}$-algebra. The following postulates, of an algebraic or operatorial nature, are needed for the reconstruction of the manifold, spin (or $\left.\operatorname{spin}^{c}\right)$ structure and Riemannian metric.

(1) Dimension: For some $p \in\{1,2,3, \ldots\}$, the operator $\langle\mathcal{D}\rangle^{-1}:=\left(1+\mathcal{D}^{2}\right)^{-1 / 2}$ lies in $\mathcal{L}^{p, \infty}$ and $\langle\mathcal{D}\rangle^{-p} \in \mathcal{L}^{1, \infty}$ has positive Dixmier traces: $\operatorname{Tr}_{\omega}\langle\mathcal{D}\rangle^{-p}>0$ for all $\omega$.

When $p$ is even, $\mathcal{H}$ is $\mathbb{Z}_{2}$-graded by a selfadjoint unitary $\Gamma$ commuting with $\mathcal{A}$ and anticommuting with $\mathcal{D}$. When $p$ is odd, $\mathcal{H}$ is ungraded and we put $\Gamma=1$ for convenience.

(2) Regularity: $(\mathcal{A}, \mathcal{H}, D)$ is $Q C^{\infty}$ in the sense of [1]: if $\delta(x):=[|\mathcal{D}|, x]$ for $x \in \mathcal{B}(\mathcal{H})$, then $\mathcal{A} \cup[\mathcal{D}, \mathcal{A}] \subseteq \bigcap_{m=1}^{\infty}$ Dom $\delta^{m}$. Moreover, $\mathcal{A}$ is complete in the locally convex topology given by the seminorms $q_{m}(a):=\left\|\delta^{m} a\right\|$ and $q_{m}^{\prime}(a):=$ $\left\|\delta^{m}([\mathcal{D}, a])\right\|$, for $m=0,1,2, \ldots$

(3) Finiteness: The prehilbert space $\mathcal{H}_{\infty}=\bigcap_{m \geq 1}$ Dom $\mathcal{D}^{m}$ is a finitely generated projective $\mathcal{A}$-module. 
(4) Absolute continuity: If $a>0$ in $\mathcal{A}$, then $\operatorname{Tr}_{\omega} a\langle\mathcal{D}\rangle^{-p}>0$ for all $\omega$.

(5) First order: $[[\mathcal{D}, a], b]=0$ for $a, b \in \mathcal{A}$.

(6) Orientability: There is a Hochschild $p$-cycle

$$
\mathbf{c}=\sum_{\alpha=1}^{n} a_{\alpha}^{0} \otimes a_{\alpha}^{1} \otimes \cdots \otimes a_{\alpha}^{p} \in Z_{p}(\mathcal{A}, \mathcal{A})
$$

which is represented on $\mathcal{H}$ by $\Gamma$, that is,

$$
\pi_{\mathcal{D}}(\mathbf{c}) \equiv \sum_{\alpha=1}^{n} a_{\alpha}^{0}\left[\mathcal{D}, a_{\alpha}^{1}\right] \ldots\left[\mathcal{D}, a_{\alpha}^{p}\right]=\Gamma .
$$

(7) Closedness: $\operatorname{Tr}_{\omega}\left(\Gamma\left[\mathcal{D}, a_{1}\right] \ldots\left[\mathcal{D}, a_{p}\right]\langle\mathcal{D}\rangle^{-p}\right)=0$ for all $a_{1}, \ldots, a_{p} \in \mathcal{A}$.

(8) Spin ${ }^{c}$ or Morita equivalence: The $C^{*}$-module completion of $\mathcal{H}_{\infty}$ is a Morita equivalence bimodule between $A$ and the norm completion of the algebra $\mathcal{C}_{\mathcal{D}}(\mathcal{A})$ generated by $\mathcal{A}$ and $[D, \mathcal{A}]$.

(9) Spin or reality: There is an antiunitary operator $J$ on $\mathcal{H}$ such that $J a^{*} J^{-1}=a$ for all $a \in \mathcal{A}, J^{2}= \pm 1, J \mathcal{D} J^{-1}= \pm \mathcal{D}$ and $J \Gamma J^{-1}= \pm \Gamma$ if $p$ is even, with the same signs (depending only on $p \bmod 8$ ) as occur when $\mathcal{A}=C^{\infty}(M)$ with $M$ a $p$-dimensional spin manifold, $\mathcal{D}$ is a Dirac operator and $J$ is the charge conjugation.

(10) Connectivity: There is an orthogonal family of projectors $p_{j} \in \mathcal{A}$ such that $\operatorname{Id}_{\mathcal{A}}=\sum_{j} p_{j}$, and $a \in \mathcal{A}$ with $[\mathcal{D}, a]=0$ if and only if $a=\sum_{j} \lambda_{j} p_{j}$ for some $\left\{\lambda_{j}\right\} \subset \mathbb{C}$.

For a detailed discussion of how each of these conditions contributes to the reconstruction of the smooth manifold $M$, we refer to [4]. For instance, the orientability condition plays a role in ensuring that all operators appearing under Dixmier traces above are 'measurable', which means that it does not matter which Dixmier trace is used. Another point to note is that, by the Serre-Swan theorem, there is a complex vector bundle $S \rightarrow M$ (that turns out to be the spinor bundle) for which $\mathcal{H}_{\infty} \subset \Gamma(M, S)$, the latter being the $A$-module of continuous sections of $S$.

Naturally, the conclusion that $M$ is a smooth manifold is rather strong and one may wonder whether some 'almost-manifolds' could also be allowed. For instance, one can build a spectral triple on an orbifold, satisfying many, but not all, of the above postulates. While one normally uses Lie groupoids [3] to describe orbifolds, which entails noncommutative algebras, one could construct a spectral triple on a quotient space $M / G$ using the algebra $\mathcal{A}=C^{\infty}(M)^{G}$ of smooth invariant functions, which satisfies many of the properties listed above. We next show that this attempt fails, precisely on account of the orientability condition.

\section{A $G$-invariant spectral triple}

Consider the following example of a commutative spectral triple: let $(M, g)$ be a compact smooth $p$-dimensional manifold without boundary, gifted with a Riemannian 
metric $g$ and a spin structure (so that, in particular, $M$ is orientable), and let $G$ be a finite group acting by isometries on $M$. We suppose that $M$ is connected; if not, it has finitely many components and we may restrict our attention to the subgroup of $G$ that preserves a given component. Assume that this action lifts to an action of $G$ on the spinor bundle $S \rightarrow M$, for which the Dirac operator $D_{g}$ is $G$-invariant. Now let $\mathcal{A}:=C^{\infty}(M)^{G}$ be the algebra of $G$-invariant smooth functions on $M, \mathcal{H}:=L^{2}(M, S)^{G}$ be the Hilbert space of $G$-invariant $L^{2}$-spinors, and let $\mathcal{D}$ be the restriction of $D_{g}$ to a selfadjoint operator on $\mathcal{H}$. Then $(\mathcal{A}, \mathcal{H}, \mathcal{D})$ is a spectral triple over a commutative unital algebra.

(It might happen that an isometric action of $G$ on $M$ lifts only to that of an extension $\widetilde{G}$ of $G$ by $\mathbb{Z}_{2}$, acting by automorphisms of the spinor bundle. Since $\widetilde{G}$ is also a finite group, the arguments below are not materially affected by this variant, so we may as well assume that the isometric action of $G$ lifts directly to the spinor bundle.)

We claim that this example cannot satisfy the orientation condition unless $G$ acts freely on $M$.

Theorem 3.1. Let $(\mathcal{A}, \mathcal{H}, \mathcal{D})$ be the p-dimensional spectral triple $\left(C^{\infty}(M)^{G}\right.$, $\left.L^{2}(M, S)^{G}, \mathcal{D}\right)$ described above. Assume that the action of the finite group $G$ is not free. Then there is no set of functions $\left\{a_{\alpha}^{j} \in C^{\infty}(M)^{G} \mid j=0,1, \ldots, p\right.$; $\alpha=1, \ldots, n\}$, such that the orientability condition (2.1) is satisfied.

REMARK 3.2. If the action of $G$ on $M$ is free, then the orbit space $X=M / G$ is a smooth Riemannian manifold and this spectral triple may be identified with a Dirac spectral triple over $C^{\infty}(X)$. In particular, all of the defining conditions listed in [4] will hold automatically for $(\mathcal{A}, \mathcal{H}, \mathcal{D})$. However, if the action is not free, then $M / G$ is instead an orbifold. We do not need to consider this quotient space as such, but much of the standard terminology of orbifold theory is useful. We follow the notation of [3], for the most part.

PROOF. The idea of the proof is as follows. If the action of $G$ is not free, then there is at least one point $x \in M$ for which the stabilizer subgroup $G_{x}$ is nontrivial. The differential $d g$ of $g \in G_{x}$ acts as an orthogonal transformation of the cotangent bundle $T_{x}^{*} M$. We show that the differential $d f$ of any invariant function $f \in C^{\infty}(M)^{G}$ lies in the subspace fixed by $d g$ for any such $g$. Since this subspace is of strictly lower dimension than $p$, there are not enough invariant functions to construct a $p$-dimensional volume form at $x$. We finish the proof by relating this defect to the failure of the orientability condition.

The isotropy subgroup of $x \in M$ is $G_{x}:=\{h \in G \mid h \cdot x=x\} \leq G$, and the fixed set of $h \in G$ is $\Sigma_{h}:=\{x \in M \mid h \cdot x=x\} \subset M$. The singular locus is 


$$
\Sigma_{G}:=\bigcup_{h \neq 1} \Sigma_{h}=\left\{x \in M \mid G_{x} \neq 1\right\}
$$

so that $G$ acts freely on its complement. The exponential map at $x \in M$ coming from the metric $g$ gives a diffeomorphism $\exp _{x}: U \rightarrow W$ from a small open neighbourhood of $0 \in T_{x} M$ to a neighbourhood $W$ of $x$ in $M$. If $h \in G$, then $d h_{x}$ is an orthogonal linear transformation from $T_{x} M$ to $T_{h \cdot x} M$, such that

$$
\exp _{h \cdot x} \circ d h_{x}=h \circ \exp _{x}: U \rightarrow h \cdot W .
$$

When $h \in G_{x}$, we may take $W$ to be $G_{x}$-invariant and may assume that each $d h_{x}$ preserves $U$, so that

$$
\exp _{x} \circ d h_{x}=h \circ \exp _{x}: U \rightarrow W
$$

in that case. Note [3, Lemma 2.10] that if $h \in G_{x}$, then $\left\{y \in \Sigma_{h} \mid d h_{y}=1\right.$ on $\left.T_{y} M\right\}$ is both closed and open in $M$ by (3.1a), so that if $M$ is connected, then $h \mapsto d h_{x}$ : $G_{x} \rightarrow \operatorname{Aut}\left(T_{x} M\right)$ is injective for all $x \in M$. Together with (3.1b), this shows that $\Sigma_{h}$ has empty interior if $h \neq 1$. Thus, the singular locus $\Sigma_{G}$ is a closed subset of $M$ with empty interior.

By transposition, each $d h_{x}$ acts on the cotangent spaces, taking $T_{h \cdot x}^{*} M$ to $T_{x}^{*} M$, again orthogonally with respect to the transposed metric $g^{-1}$. In other words, $d h$ has a right action on covectors.

Any $G$-invariant function $f$ equals its average over any cyclic subgroup of $G$ :

$$
f(x)=\frac{1}{|h|} \sum_{j=0}^{|h|-1} f\left(h^{j} \cdot x\right),
$$

where $|h|$ denotes the order of $h$. If $h \in G_{x}$, taking commutators with the Dirac operator gives

$$
[\mathcal{D}, f](x)=c(d f(x))=\frac{1}{|h|} \sum_{j=0}^{|h|-1} c\left(d f(x) \triangleleft d h_{x}^{j}\right),
$$

where $c$ denotes Clifford multiplication and $\triangleleft$ denotes the right action of $d h_{x}$ on $T_{x}^{*} M$. Note that (3.1b) implies that the notation $d h_{x}^{j}$ is unambiguous: $\exp _{x} \circ\left(d h_{x}\right)^{j}=h^{j} \circ$ $\exp _{x}=\exp _{x} \circ\left(d h^{j}\right)_{x}$, so that $\left(d h_{x}\right)^{j}=\left(d h^{j}\right)_{x}$ on $T_{x} M$.

Let $x \in \Sigma_{G}$. The Cartan-Dieudonné theorem, showing that every orthogonal transformation on $T_{x} M$ is the product of at most $p$ reflections, allows us to choose, for each $h \in G_{x}$, an orthonormal basis for $T_{x} M$ such that

$$
d h_{x}=\operatorname{diag}\left(R_{\theta_{1}}, R_{\theta_{2}}, \ldots, R_{\theta_{k}}, \pm 1, \ldots, \pm 1\right),
$$

where each $R_{\theta_{j}}$ is a two-by-two rotation matrix (which itself is a product of two reflections). 
Consider first the case where $G$ acts on $M$ by orientation-preserving isometries, so that each $d h_{x}$ is a rotation in $S O\left(T_{x} M\right)$. Then we can write

$$
[\mathcal{D}, f](x)=c(d f(x))=c(u)+c\left(v_{1}\right)+\cdots+c\left(v_{k}\right),
$$

where $u \in T_{x}^{*} M$ is invariant under $d h_{x}$ and $v_{r} \triangleleft d h_{x}=R_{-\theta_{r}} v_{r}$, for $r=1, \ldots, k$. Then

$$
\begin{aligned}
{[\mathcal{D}, f](x) } & =\frac{1}{|h|} \sum_{j=0}^{|h|-1} c\left(d f(x) \triangleleft d h_{x}^{j}\right) \\
& =\frac{1}{|h|} \sum_{j=0}^{|h|-1} c\left(u+R_{-\theta_{1}}^{j} v_{1}+\cdots+R_{-\theta_{k}}^{j} v_{k}\right) \\
& =c(u)+\frac{1}{|h|} \sum_{r=1}^{k}\left(\sum_{j=0}^{|h|-1} R_{-\theta_{r}}^{j}\right) v_{r}=c(u),
\end{aligned}
$$

since $\sum_{j=0}^{|h|-1} R_{-\theta_{r}}^{j}=0$ : that follows since the order of each rotation $R_{-\theta_{r}}$ divides $|h|$.

Hence, if $f$ is any $G$-invariant function, $[\mathcal{D}, f](x)$ is fixed by the rotation $d h_{x}$ and so it is (Clifford multiplication by) a covector perpendicular to all of the planes of rotation of $h$.

Therefore, if $G_{x} \neq 1$ and if $a_{0}, a_{1}, \ldots, a_{p}$ are $G$-invariant functions, then $a_{0}\left[\mathcal{D}, a_{1}\right] \ldots\left[\mathcal{D}, a_{n}\right]$ has vanishing skewsymmetrization at $x$ :

$$
\frac{1}{p !} \sum_{\sigma \in S_{p}}(-1)^{\sigma} \sum_{\alpha} a_{0}(x)\left[\mathcal{D}, a_{\sigma(1)}\right](x) \ldots\left[\mathcal{D}, a_{\sigma(p)}\right](x)=0,
$$

since, under the usual linear isomorphism $\mathbb{C} \ell\left(T_{x}^{*} M, g_{x}\right) \simeq \Lambda^{\bullet} T_{x}^{*} M$, the left-hand side corresponds to a $p$-covector in $\Lambda^{p} V$, where $V=\bigcap_{h \in G_{x}} \operatorname{ker}\left(\triangleleft d h_{x}\right)$ has dimension less than $p$.

In particular, on inserting the entries of the Hochschild $p$-cycle (2.1a) in equation (3.2), we obtain

$$
\Gamma^{\prime}(x)=\frac{1}{p !} \sum_{\sigma \in S_{p}}(-1)^{\sigma} \sum_{\alpha} a_{\alpha}^{0}(x)\left[\mathcal{D}, a_{\alpha}^{\sigma(1)}\right](x) \ldots\left[\mathcal{D}, a_{\alpha}^{\sigma(p)}\right](x)=0,
$$

for all $x \in \Sigma_{G}$. We have written $\Gamma^{\prime}$ for the skewsymmetrized version of the represented cycle (2.1b). Here $\Gamma^{\prime}$ may be regarded as an endomorphism of the $\mathcal{A}$-module $\mathcal{H}_{\infty}$ and thereby as a continuous section in $\Gamma(M$, End $S)$.

Next consider the case where some $G_{x}$ contains a reflection.

Let $x \in \Sigma_{G}$ and $h \in G_{x}$ be such that $d h_{x}$ is a reflection in the hyperplane $v^{\perp}$, for some unit covector $v \in T_{x}^{*} M$. Thus, if $f$ is $G$-invariant, then

$$
\begin{aligned}
c(d f(x)) & =[\mathcal{D}, f](x)=\frac{1}{2}\left(c(d f(x))+c\left(d f(x) \triangleleft d h_{x}\right)\right) \\
& =\frac{1}{2} c(d f(x))+\frac{1}{2}\left(c(d f(x))-2 g_{x}(v, d f(x)) c(v)\right) \\
& =c(d f(x))-g_{x}(v, d f(x)) c(v) .
\end{aligned}
$$


Thus $g_{x}(v, d f(x))=0$, so $d f(x)$ is a covector in the hyperplane $v^{\perp}$. Again, any $p$ such covectors have null exterior product, so that $\Gamma^{\prime}(x)=0$ in this case also.

Suppose now that there are finitely many $G$-invariant functions $\left\{a_{\alpha}^{j} \mid j=0, \ldots, p\right.$, $\alpha=1, \ldots, n\}$ such that $\mathbf{c}$ as given by (2.1a) is a Hochschild $p$-cycle, and that equation $(2.1 b)$ holds with $\Gamma$ being proportional to Clifford multiplication by the volume element, as happens when $\mathcal{D}$ is the Dirac operator. By using local orthonormal bases of 1-forms, one can check that the skewsymmetrization of this section of the Clifford algebra bundle is, up to a constant, Clifford multiplication by the Riemannian volume form $\operatorname{vol}_{g}$ on $M$. As such, the skewsymmetrization is nowhere vanishing and the norm of $\Gamma^{\prime}(y) \in$ End $S_{y}$ has a positive lower bound. Since we have shown that for $G$-invariant functions there is always a neighbourhood of the singular locus $\Sigma_{G}$ on which $\left\|\Gamma^{\prime}(y)\right\|$ is smaller than any such lower bound, the orientability condition cannot be satisfied.

The argument extends to any compact orbifold, since these are locally of the form $M / G$ with $G$ finite. Indeed, such an orbifold $X$ is a topological space that may be covered by finitely many orbifold charts [3], each of the form $U / G$ where $U$ is an open connected subset of $\mathbb{R}^{p}$ and $G$ is a finite group of diffeomorphisms of $U$; one may impose a Riemannian metric on $U$ for which $G$ acts by isometries. Overlapping charts may use different finite groups, but all are linked by compatibility homomorphisms. The singular locus of the orbifold is the union of finitely many sets $\Sigma_{G}$ in each such chart, and the above argument shows that the candidate for a volume form, if it comes from a $G$-invariant $p$-form on each $U$, must always vanish on the singular locus, and therefore cannot satisfy (2.1b).

While the argument is local, it is not clear that for a general orbifold we have a good candidate for a (commutative) algebra of functions: this is highly dependent on the pasting conditions [3]. When dealing with quotients $M / \Gamma$, where $\Gamma$ is an infinite discrete group acting locally freely on $M$ (that is, all isotropy subgroups are finite), there is such a candidate algebra but no simple method of suitably encoding the local freedom. It is therefore more appropriate to employ the groupoid algebra in these situations.

\section{Acknowledgements}

We thank Gianni Landi for first asking us about the admissibility of orbifolds as commutative geometries. Later, others have raised the same question. JCV is grateful to Ryszard Nest for kind hospitality at the University of Copenhagen.

\section{References}

[1] A. L. Carey, J. Phillips, A. Rennie and F. A. Sukochev, 'The Hochschild class of the Chern character for semifinite spectral triples', J. Funct. Anal. 213 (2004), 111-153.

[2] A. Connes, 'Gravity coupled with matter and foundation of noncommutative geometry', Commun. Math. Phys. 182 (1996), 155-176. 
[3] I. Moerdijk and J. Mrčun, Introduction to foliations and Lie groupoids (Cambridge University Press, Cambridge, 2003).

[4] A. Rennie and J. C. Várilly, 'Reconstruction of manifolds in noncommutative geometry', Preprint arXiv:math/0610418 [math.OA], Copenhagen, 2006.

ADAM RENNIE, Institute for Mathematical Sciences, University of Copenhagen, Universitetsparken 5, DK-2100 Copenhagen, Denmark

and

Department of Mathematics, Australian National University, Canberra, ACT, 0200, Australia

e-mail: adam.rennie@maths.anu.edu.au

JOSEPH C. VÁRILLY, Departamento de Física Teórica I, Universidad Complutense, Madrid 28040, Spain

and

Departamento de Matemáticas, Universidad de Costa Rica, 2060 San José, Costa Rica e-mail: varilly@cariari.ucr.ac.cr 